\section{Increased body mass index and reduced efficacy of oral emergency contraception: how many women might be affected?}

Oral emergency contraception (EC) is available as levonorgestrel and ulipristal acetate. In England, EC is available through a variety of settings and can be obtained without a prescription. In 2014, evidence from clinical studies prompted a review of available evidence by the European Medicine Agency's Committee for Medicinal Products for Human Use to assess whether increased bodyweight reduces the efficacy of EC. The review concluded that the available data were inconclusive. ${ }^{12}$ However, if future research were to substantiate a relationship of reduced EC efficacy in women with higher bodyweight, it would be important to understand the potential impact at a population level. We used data from the Health Survey for England (HSE) $2010^{3}$ to explore this.

HSE is an annually conducted, nationally representative, probabilistic household survey of the general population resident in England. Details of HSE methodology are reported elsewhere. ${ }^{4} 5$ Demographic data are collected using face-to-face interviews and self-completed questionnaire booklets. Bodyweight is measured at a nurse visit. In 2010, the survey incorporated questions regarding sexual behaviour and contraceptive use, including EC use in

Table 1 Percentage of women who reported oral emergency contraception use in the last year (sexually experienced women aged 16-44 years)

\begin{tabular}{lc}
\hline Women & Used EC in last year $(95 \% \mathrm{Cl})$ \\
\hline Overall & $9 \%(7-11 \%)$ \\
By age group (years) \\
$16-24$ & $20 \%(16-26 \%)$ \\
$25-34$ & $8 \%(6-11 \%)$ \\
$35-44$ & $3 \%(2-5 \%)$ \\
\hline
\end{tabular}

$\mathrm{Cl}$, confidence interval; $\mathrm{EC}$, emergency contraception.

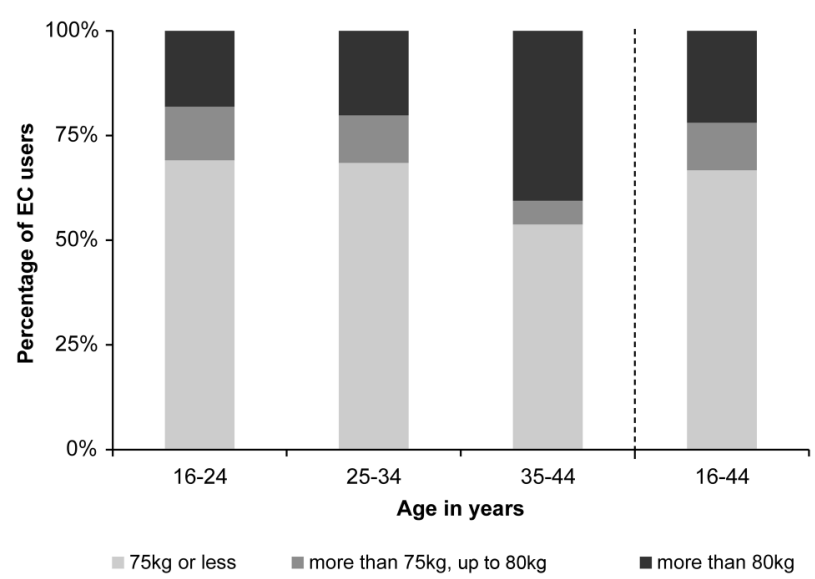

Figure 1 Bodyweight of emergency contraception (EC) users by age group (sexually experienced women aged 16-44 years).

the 12 months preceding the interview. We estimated EC use in 1508 sexually experienced (reporting at least one sexual partner over the lifetime to the date of the interview) $16-44$ year-old women by bodyweight. Data were analysed in Stata V.12.1 accounting for weighting, clustering and stratification of the data.

EC use in the last year was reported by $9 \%[95 \%$ confidence interval (CI) $7-11 \%$ ] of all sexually experienced 16-44 year-old women and was more frequently reported among younger women (Table 1). Among those who reported EC use in the last year, 33\% (95\% CI 25-43\%) had a bodyweight $>75 \mathrm{~kg}$ and 22\% (95\% CI 15-31\%) had a bodyweight $>80 \mathrm{~kg}$ (Figure 1).

Current guidance advises EC use regardless of bodyweight, ${ }^{1}$ although available data remain inconclusive with regard to the relationship with EC efficacy. ${ }^{1}{ }^{2}$ Our analysis demonstrates that EC could be less effective in a substantial proportion of current EC users if efficacy were found to be lower among those with higher bodyweight. If future provision of EC is reliant on some measure of bodyweight, this would have serious implications in the delivery of EC, especially in settings where the threshold for access to EC is low, such as pharmacies. Younger women would be particularly affected given the higher rates of EC use in this group. Research is urgently needed to determine the relationship between bodyweight and EC efficacy.

\section{Sarah C Woodhall*, PhD}

Principal Scientist, National Infection Service, Public Health England (PHE), London, UK; sarah.woodhall@phe.gov.uk

\section{Catherine M Lowndes, PhD}

Consultant Epidemiologist, National Infection Service, PHE, London, UK; cmlowndes@googlemail.com

\section{Rachel Craig, BA}

Group Head, NatCen Social Research, London, UK; rachel.craig@natcen.ac.uk

\section{Jennifer S Mindell, PhD}

Reader in Public Health, Research Department of Epidemiology and Public Health, UCL, London, UK; j.mindell@ucl.ac.uk

\section{Dame Jane Anderson, PhD, FRCP}

Consultant Physician and Advisor, Health and Wellbeing Directorate, PHE, London, UK; jane.anderson@phe.gov.uk

\section{Kate A Guthrie, FRCOG, FFSRH}

Clinical Expert, Sexual \& Reproductive Health, Health and Wellbeing Directorate, PHE, London, UK; kate.guthrie@phe.gov.uk

\section{Dame Anne M Johnson, MD, FMedSci, FRCP, FFPH, FRCGP}

Professor of Infectious Disease Epidemiology, Research Department of Infection and Population Health, UCL, London, UK; anne.johnson@ucl.ac.uk

\section{Anthony Nardone, PhD}

Consultant scientist, National Infection Service, Public Health England, London, UK

Health and Wellbeing Directorate, Public Health England, London, UK; anthony.nardone@phe.gov.uk *Corresponding author

Competing interests Kate Guthrie has previously advised and given lectures for the manufacturer of ellaOne ${ }^{\circledR}$ (HRA Pharma).

Authors' note These findings were presented as a poster presentation at the 2015 Lancet Public Health Science Conference. $^{6}$ 
J Fam Plann Reprod Health Care 2016;42:234-235. doi:10.1136/jprhc-2016-101464

\section{REFERENCES}

1 European Medicines Agency. Levonorgestrel and ulipristal remain suitable emergency contraceptives for all women, regardless of bodyweight. 2014. http://www.ema.europa.eu/ docs/en_GB/document_library/Referrals_ document/Emergency_contraceptives_31/ WC500176381.pdf [accessed 19 October 2015].

2 Faculty of Sexual \& Reproductive Healthcare. Statement from the Clinical Effectiveness Unit on labelling of emergency contraception in Europe: body weight, body mass index (BMI) and efficacy. 2014. https://www.fsrh. org/documents/cec-ceu-statement-ec-bmi-wtjul-14/cec-ceu-statement-ec-bmi-wt-jul-14.pdf [accessed 19 October 2015].

3 NatCen Social Research and Royal Free and University College Medical School. Department of Epidemiology and Public Health. Health Survey for England, 2010 [computer file]. 3rd Edition. Colchester, Essex: UK Data Archive [distributor], January 2015. SN: 6986, doi:10.5255/UKDA-SN-6986-3.

4 Mindell J, Biddulph JP, Hirani V, et al. Cohort profile: the health survey for England. Int J Epidemiol 2012;41:1585-1593.

5 Health \& Social Care Information Centre. Health Survey for England 2012. Volume 2: Methods and Documentation. 2013. http://www.hscic.gov.uk/ catalogue/PUB13218/HSE2012-Methodsand-docs.pdf [accessed 19 October 2015].

6 Woodhall SC, Lowndes CM, Craig R, et al. Emergency hormonal contraception and bodyweight: analysis of data from the Health Survey for England, 2010. Poster presentation at the 2015 Lancet Public Health Science Conference, London, UK, 13 November 2015. 